TITLE:

\title{
Numerical Analysis on Gas Separator with Thermal Transpiration in Micro Channels
}

\author{
$\operatorname{AUTHOR}(\mathrm{S})$ :
}

Sugimoto, Hiroshi; Hibino, Masaya

\section{CITATION:}

Sugimoto, Hiroshi ... [et al]. Numerical Analysis on Gas Separator with Thermal Transpiration in Micro Channels. AIP Conference Proceedings: 28th International Symposium on Rarefied Gas Dynamics 2012 2012, 1501: 794-801

\section{ISSUE DATE:}

2012-11-27

URL:

http://hdl.handle.net/2433/187980

\section{RIGHT:}

(c) 2012 American Institute of Physics. This article may be downloaded for personal use only. Any other use requires prior permission of the author and the American Institute of Physics. 


\title{
Numerical Analysis on Gas Separator with Thermal Transpiration in Micro Channels
}

\author{
Hiroshi Sugimoto and Masaya Hibino
}

Department of Aeronautics and Astronautics, Kyoto University, Kyoto 606-8501, Japan

\begin{abstract}
A gas separator that can induce a large variation of mole fraction by a small temperature difference in a single membrane is proposed. The separator makes use of two effects: The first effect is the thermal transpiration through microchannels in the membrane; the second is the accumulation of the effect of micro-channels in the counter flow of gas mixture that flows around the membrane. The numerical results show that small gas separation effects of numerous micro-channels are accumulated to induce a large variation of the mole fraction along the membrane. The numerical calculation is carried out by the direct simulation Monte Carlo (DSMC) method and a fluid model based on the mass conservation which is shown to be able to simulate the DSMC result. The performance of the device is investigated for several temperature differences between the two sides of the membrane. The relation to the membrane gas separation by Knudsen diffusion is also discussed.
\end{abstract}

Keywords: thermal transpiration, membrane gas separation, molecular gas dynamics, Knudsen pump, Knudsen diffusion PACS: $47.56 .+\mathrm{r}, 82.39 . \mathrm{Wj}, 28.60 .+\mathrm{s}, 51.10 .+\mathrm{y}, 47.45 .-\mathrm{n}$

\section{INTRODUCTION}

Recent membrane technology have enabled the mass production of various polymeric membranes with through holes of sub-micron size. The diameter of the hole is comparable to the size of the mean free path of gas molecules in air under atmospheric pressure, hence various rarefied gas effects are expected in the holes. One of the applications of these rarefied gas effects is the Knudsen pump, a non-mechanical pump driven by the thermal transpiration of the rarefied gas. Gupta and Gianchandani recently reported that a Knudsen pump of dime-size can induce a flow at a speed of $1 \mathrm{~cm} / \mathrm{min}$ through a mixed cellulose ester membrane and create $1 \mathrm{kPa}$ of pressure difference by blocking the flow [1]. Another well-known application may be the membrane gas separation [2] by Knudsen diffusion, that is, smaller molecules travel faster than larger molecules in permeate flows. Several studies have been carried out on the integration of these subjects: thermally driven pumps and gas mixture separation. In Refs. [3, 4], we found that a difference of mole fraction of mixture is induced between the two ends of a thermally driven pump when both ends are choked up to form a closed system. The difference of mole fraction is confirmed experimentally in [5]. In our previous paper [6], we propose an open gas separator system, which continuously produces concentrated gases from a feed gas. The results of DSMC simulations show that the thermal transpiration has an influence on the mole fraction of continuous flow of feed gas. The variation of mole fraction obtained in [6] is, however, very small despite a large temperature difference is used in the device. In view of the fabrication of the device, the large temperature difference is impossible since the maximum operating temperatures of polymeric membranes are usually low, e.g., around 400 $\mathrm{K}$. The purpose of the present paper is to find a method of obtaining a large variation of mole fraction with thermal transpiration induced by a small temperature variation in a membrane.

\section{OPERATION OF THE GAS SEPARATOR}

The schematic of the gas separator in this paper is shown in Fig. 1(a). The separator consists of a porous membrane with micro-channels, and two channels ( $\mathrm{F}$ and $\mathrm{R}$ ) along the both sides of it. The channel $\mathrm{F}$ is connected to channel $\mathrm{R}$ at one end to form a U-bend channel. The feed gas, a binary mixture of smaller gas A and larger gas $\mathrm{B}$, enters the device at the other end of $\mathrm{F}$ and flows through the U-bend channel toward the outlet at channel R. At the same time, a molecular flow, the speed of which generally depends on species of molecule, is induced through each microchannel in the membrane. This results in a variation of mole fraction in channels $\mathrm{F}$ and $\mathrm{R}$ around the individual micro-channels; an increase of the mole fraction in channel $\mathrm{F}$ leads to a decrease of that in $\mathrm{R}$ and vise versa, assuming that the mole fractions in F and R are close enough. The flow in U-bend channel convects the variations of the mole 


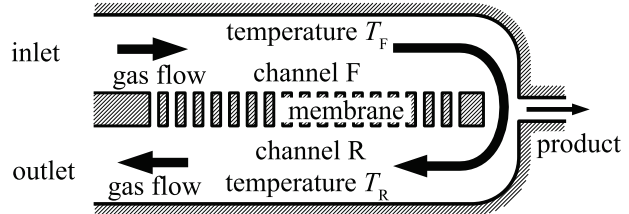

(a)

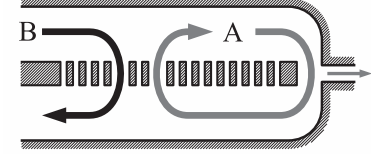

(b)

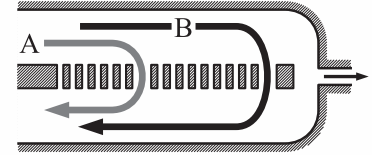

(c)

FIGURE 1. (a) The schematic of the gas separator. (b) Schematic of flows of smaller molecule A and larger molecule B for $T_{\mathrm{F}} / T_{\mathrm{R}}>1$. (c) Schematic of flows for $T_{\mathrm{F}} / T_{\mathrm{R}} \leq 1$.

fraction downstream, accumulating the effects of many micro-channels. Due to the present counter flow setup, the gradients of the mole fraction along the membrane in $\mathrm{F}$ and $\mathrm{R}$ can be almost the same. As the result, the difference of mole fraction between $\mathrm{F}$ and $\mathrm{R}$ is kept so small that the molecular flows in micro-channels are not disturbed. This counter flow setup is the main improvement on our previous work [6], where a parallel flow setup in F and R is applied. The product of the device is a small amount of the gas from the connection of $F$ and $R$.

Now we discuss the gas flow in individual micro-channels. It is induced by the gradients of pressure, temperature, and mole fraction. The pressure in channel $\mathrm{F}$ is inevitably larger than that in $\mathrm{R}$, and we can control their difference with that between the inlet and outlet of the device. The temperature $T_{\mathrm{F}}$ of channel $\mathrm{F}$ and $T_{\mathrm{R}}$ of $\mathrm{R}$ can be set freely. In this section, we estimate the behavior of mole percentage $\chi$ of smaller gas A, defined by

$$
\chi=100 n^{\mathrm{A}} / n, \quad\left(n^{\mathrm{A}}: \text { number density of A molecules; } n: \text { number density of mixture }\right)
$$

from the flows induced by the pressure and temperature gradients along the micro-channel.

First we consider the case where the thermal transpiration occurs in the direction of $\mathrm{R} \rightarrow \mathrm{F}$, that is, $T_{\mathrm{F}} / T_{\mathrm{R}}>1$, and its flow speed is similar to that of pressure driven flow in the direction of $F \rightarrow R$. Since the main parts of the flows cancel each other out, the species dependence of the flows becomes important. The common property of these flows is that the smaller A-gas flows faster than B-gas. The speed ratios are commonly $\sqrt{m^{\mathrm{B}} / m^{\mathrm{A}}}\left(m^{\alpha}\right.$ : mass of $\alpha$ molecule) in Knudsen limit. In the continuum limit, the flow speed of pressure driven flow does not vanish, and the speed ratio approaches unity there. On the other hand, the thermal transpiration vanishes in this limit, keeping nearly constant speed ratio $\sqrt{m^{\mathrm{B}} / m^{\mathrm{A}}}$. Therefore, at intermediate Knudsen numbers, it is possible that A-molecules flow in the direction $\mathrm{R} \rightarrow \mathrm{F}$ while the same number of $\mathrm{B}$-molecules flow $\mathrm{F} \rightarrow \mathrm{R}$ under appropriate gradients of pressure and temperature along the micro-channel [6]. Hereafter this state is referred as "molecular exchange" in this paper. If the pressure gradient is larger (smaller) than the appropriate value, the flows are accelerated in the direction of $\mathrm{F} \rightarrow \mathrm{R}$ $(\mathrm{R} \rightarrow \mathrm{F})$. Nevertheless A-molecules have larger velocity in $\mathrm{R} \rightarrow \mathrm{F}$ direction. The behavior of the gases in the separator device under this condition is shown exaggeratedly in Fig. 1(b); The B-gas is ejected from the device by the flow $\mathrm{F} \rightarrow \mathrm{R}$, while some part of A-gas forms a circulation around the bottom of U-bend channel by the flow $\mathrm{R} \rightarrow \mathrm{F}$ through the membrane. Then the value of $\chi$ of product gas is larger than that in the feed gas.

For other cases, $T_{\mathrm{F}} / T_{\mathrm{R}}<1$ or the thermal transpiration is very weak, both of molecules $\mathrm{A}$ and $\mathrm{B}$ flow in the direction of $\mathrm{F} \rightarrow \mathrm{R}$. The speed of the flow is larger for A-gas. Figure 1(c) explains the behavior of the gases under this condition; A-gas is ejected from the device earlier, and the value of $\chi$ of the product gas becomes lower than that of the feed gas. The thermal transpiration flow in $\mathrm{F} \rightarrow \mathrm{R}$ direction will intensify this effect.

\section{METHODS OF SOLUTION}

The numerical model of the gas separator is shown in Fig. 2. The model is 2D in $X_{1}-X_{2}$ space, and the U-bend channel is replaced with two channels $\mathrm{R}$ (temperature $T_{\mathrm{R}}$ ) and $\mathrm{F}$ (temperature $T_{\mathrm{F}}$ ) of width $D$ parallel to $X_{1}$-axis. The membrane is modeled as an array of $m$ micro-channels of width $d$ and length $t$ with a constant spacing $d$. The temperature of the walls of micro-channels vary linearly from $T_{\mathrm{R}}$ to $T_{\mathrm{F}}$. We will number the micro-channels $i=1,2, \ldots, m$ in ascending order of their position $X_{1}$. The micro-channel $i=1$ is located at $0<X_{1}<d$, and the channels $\mathrm{F}$ and $\mathrm{R}$ starts at $X_{1}=-L_{-}$and ends at $X_{1}=L+L_{+}$, where $L=2 d m$. The feed gas (pressure $p_{\mathrm{F}}$, temperature $T_{\mathrm{F}}$, and mole percentage $\chi_{\mathrm{F}}$ of A-gas) enters channel $\mathrm{F}$ at $X_{1}=-L_{-}$. It exits channel $\mathrm{F}$ at $X_{1}=L+L_{+}$with mole percentage $\chi_{\mathrm{FL}}$. In the simplest case with no product gas, all of the gas is refluxed into channel $\mathrm{R}$ at $X_{1}=L+L_{+}$, after regulating the temperature to $T_{\mathrm{R}}$ with some pressure drop $p_{\mathrm{D}}$. We assume that in this reflux process, the mole percentage is kept constant at $\chi_{\mathrm{FL}}$. 


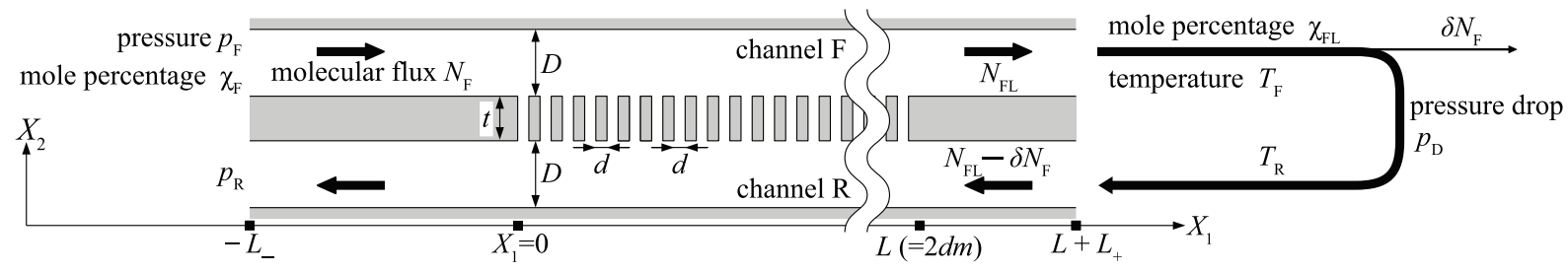

FIGURE 2. Numerical model of the gas separator.

The gas exits the device at $X_{1}=-L_{-}$from channel R. The pressure, temperature, and number density of the mixture at the outlet are $p_{\mathrm{R}}, T_{\mathrm{R}}$ and $n_{\mathrm{R}}\left(=p_{\mathrm{R}} / \kappa T_{\mathrm{R}}, \kappa\right.$ : Boltzmann constant), respectively. The mean free path $\ell_{\mathrm{R}}$ of A-gas at the stationary state with number density $n_{\mathrm{R}}$ defines the Knudsen number $\mathrm{Kn}=\ell_{\mathrm{R}} / d$. Finally we introduce the production rate $\delta$ of the gas separator. The molecular flux of the product gas is denoted by $\delta N_{\mathrm{F}}$, where $N_{\mathrm{F}}$ is the molecular flux of the feed gas. This amount of molecular flux $\delta N_{\mathrm{F}}$ is removed in the reflux process described above. Please note that the value of the molecular flux $N_{\mathrm{FL}}$ in channel F at $X_{1}=L+L_{+}$differs from that of the feed gas $N_{\mathrm{F}}$.

First we analyze the behavior of the mixture in the above model by DSMC simulation by Bird [7]. The method is well-known, and we explain only the boundary conditions used in the simulation. The boundary condition on the solid wall is the diffuse reflection. The boundary conditions at both ends of $\mathrm{F}$ and $\mathrm{R}$ are the appropriate Maxwellian distribution as in our previous work [6]. That is, we use the prescribed values for pressure and temperature. For the flow velocity we assume $\left(v_{1}, v_{2}\right)=\left(C u_{\mathrm{B}}\left(X_{2}\right), 0\right)$, where $u_{\mathrm{B}}\left(X_{2}\right)$ is the appropriate quadratic distribution in $X_{2}$ direction. The value of the coefficient $C$ for each end is determined iteratively by the molecular flux averaged in a test section arranged near the corresponding end of the channels. For the values of mole percentage at inlets, we use $\chi_{\mathrm{F}}$ for channel $\mathrm{F}$ and $\chi_{\mathrm{FL}}$ for $R$. The values at outlets are determined iteratively by the averages in each test section nearby.

The advantage of the present device is the accumulation of small effects of numerous micro-channels. It is, however, difficult to carry out DSMC calculation for large systems consisting of numerous subsystems. Instead, the mass conservation can be a useful tool to estimate the overall features of large systems [8]. Hereafter we propose a practical method, based on the mass conservation, to estimate the performance of the device with reasonable computational time. The verification of the method will be given by comparing the results of DSMC and proposed method.

We pay attention only on the longitudinal variations of the partial pressures $p^{\alpha}$ of $\alpha$-gas in channel \# (\#=F, R). The temperature of the gas is assumed to be $T_{\#}$ in each channel. We divide the channel \# into small sections $\Omega_{\#(i)}$ of width $2 d$ in $X_{1}$ direction, where $i$ is the index of the micro-channel in $\Omega_{\#(i)}$. We determine the time-variation of the number of $\alpha$-molecules in $\Omega_{\#(i)}$ by the molecular fluxes to the section $\Omega_{\#(i)}$. For example, we have

$$
\left(2 d D / \kappa T_{\mathrm{F}}\right)\left(p_{\mathrm{F}(i)}^{\alpha(k+1)}-p_{\mathrm{F}(i)}^{\alpha(k)}\right) / \Delta \tau=D\left(F_{\mathrm{F}(i-1)}^{\alpha(k)}-F_{\mathrm{F}(i)}^{\alpha(k)}\right)+d q_{(i)}^{\alpha(k)} \quad(\alpha=\mathrm{A}, \mathrm{B}),
$$

for channel F, where $p_{\mathrm{F}(i)}^{\alpha(k)}$ is the partial pressure of $\alpha$-gas in $\Omega_{\mathrm{F}(i)}$ at time $\tau=k \Delta \tau, F_{\mathrm{F}(i)}^{\alpha(k)}$ is the molecular flow rate of $\alpha$-gas from $\Omega_{\mathrm{F}(i)}$ to $\Omega_{\mathrm{F}(i+1)}$ per unit time and per unit area, and $q_{(i)}^{\alpha(k)}$ is that through $i$-th micro-channel in the direction of $\mathrm{R} \rightarrow \mathrm{F}$. The above formulation can be extended to channel $\mathrm{R}$, and the parts of channels with no micro-channel. The system of difference equations can be solved with the expression of $q_{(i)}^{\alpha(k)}$ and $F_{\#(i)}^{\alpha(k)}$. According to the result by the linearized Boltzmann equation [9], the following formula can be used for the mass flow rate $q^{\alpha}$ in a micro-channel:

$$
q^{\alpha}=\bar{n} \sqrt{2 \kappa \bar{T} / m^{\mathrm{A}}}\left[N_{T}^{\alpha}\left(T^{\prime} / \bar{T}\right)+N_{p}^{\alpha}\left(p^{\prime} / \vec{p}\right)+N_{\chi}^{\alpha}\left(\chi^{\prime} / 100\right)\right] d,
$$

where $\bar{n}, \bar{T}$, and $\bar{p}$ are the representative values of number density, temperature, and pressure, respectively; the values emphasized with prime indicate the corresponding gradients along the micro-channel. The values of the nondimensional coefficients $N_{*}^{\alpha}(*=T, p$, and $\chi)$ are given by the numerical database as the function of intermolecular potential, mass ratio $\mathrm{m}^{\mathrm{B}} / \mathrm{m}^{\mathrm{A}}$, the representative values of Knudsen number $\overline{\mathrm{Kn}}$, and that for mole percentage $\bar{\chi}$. Since the values of $p^{\prime}, \chi^{\prime}, \overline{\mathrm{Kn}}$ etc. are easily approximated by $p_{\#(i)}^{\alpha(k)}$ etc., we obtain the values of $q_{(i)}^{\alpha(k)}$. On the other hand, we have no precise data for $F_{\#(i)}^{\alpha(k)}$ : they are non-vanishing fluxes that may affect the mole fraction downstream (that is, they reflects the effect of convection terms of fluid dynamic equations), and the channel walls have innumerable holes. Here, we assume that they still consist of the part of plane Poiseuille flow proportional to pressure gradient 


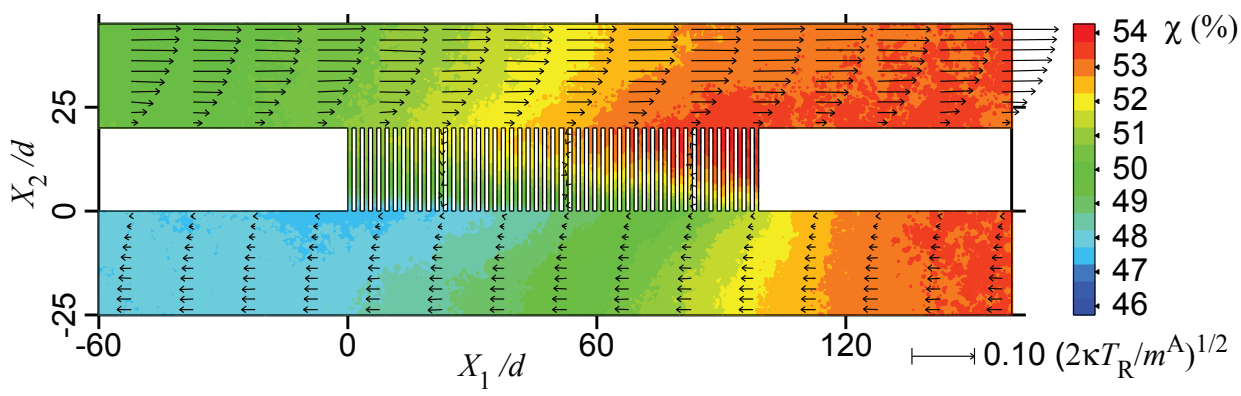

FIGURE 3. DSMC result for steady behavior of the mixture in the gas separator. The distribution of the mole percentage of smaller gas is shown by the color map, and the velocity of the mixture is shown by arrows. $T_{\mathrm{F}} / T_{\mathrm{R}}=3, \mathrm{~m}^{\mathrm{B}} / \mathrm{m}^{\mathrm{A}}=10, \chi_{\mathrm{F}}=50 \%$, $\mathrm{Kn}=0.511, m=50, t / d=20, D / d=50, p_{\mathrm{F}} / p_{\mathrm{R}}=1.329, p_{\mathrm{D}} / p_{\mathrm{R}}=0.190$, and $\delta=0.355$. The cell size of DSMC is $0.5 d \times 0.5 d$, and 200 particles are used in a cell to express the gas at number density $n_{\mathrm{R}}$.

$p^{\prime}=\mathrm{d} p / \mathrm{d} X_{1}$ and the part of diffusion proportional to $\chi^{\prime}=\mathrm{d} \chi / \mathrm{d} X_{1}$, and apply Eq. (2) (with the replacement of $d$ to $D$ ) to evaluate $F_{\#(i)}^{\alpha(k)}$ with the aid of finite difference approximation of the derivatives $p^{\prime}$ and $\chi^{\prime}$. The coefficient $N_{p}^{\alpha}$ for large values of $D / d$ may be replaced with the data by plane Poiseuille flow to save CPU time.

The difference equation Eq. (1) is of diffusion type, since $F_{\#(i)}^{\alpha(k)}$ is expressed by the difference of $p_{\#(i+1)}^{\alpha(k)}$ and $p_{\#(i)}^{\alpha(k)}$. Therefore we need two boundary conditions for each channel and gas component. These eight conditions are given by (i) total pressures $p=p^{\mathrm{A}}+p^{\mathrm{B}}$ at inlet of $\mathrm{F}\left(p=p_{\mathrm{F}}\right)$ and outlet of $\mathrm{R}\left(p=p_{\mathrm{R}}\right)$, (ii) the values of mole percentage at inlet ( $\chi_{\mathrm{F}}$ for $\mathrm{F} ; \chi_{\mathrm{FL}}$ for $\mathrm{R}$ ), (iii) the condition $\mathrm{d} \chi / \mathrm{d} X_{1}=0$ at the outlets of $\mathrm{F}$ and $\mathrm{R}$, (iv) pressure drop $p_{\mathrm{D}}$ between the outlet of $\mathrm{F}$ and inlet of $\mathrm{R}$, and (v) the relation of molecular flux at the connection of $\mathrm{F}$ and $\mathrm{R}$ as indicated in Fig. 2. Please note that $\chi_{\mathrm{FL}}$ in (ii) is determined as the solution for channel F. The above method is referred as "pipenet method" below. In the numerical calculation, we rewrite explicit form of the difference equation Eq. (1) for $p^{\alpha}$ into the implicit form for $p^{\mathrm{A}}$ and $p$ to obtain steady solution quickly with no restriction of CFL condition. In order to reduce the access to the database of $N_{*}^{\alpha}$, the values of $q_{(i)}^{\alpha(k)}$ of $M$ channels nearby are replaced by those of a representative channel.

\section{RESULTS}

\section{Comparison of the Results by DSMC and Pipenet Method}

Figure 3 shows an example of the steady state of the mixture obtained by DSMC method for feed gas concentration $\chi_{\mathrm{F}}=50 \%$. The DSMC analysis is limited to small numbers of $m$ and $D / d$ due to its computational cost; These values for the case in Fig. 3 are $m=50$ and $D / d=50$, respectively. In order to demonstrate the possibility of the gas separator with limited number of $m$, we select large values of the temperature ratio $T_{\mathrm{F}} / T_{\mathrm{R}}=3$ and mass ratio $m^{\mathrm{B}} / m^{\mathrm{A}}=10$. We assume hard-sphere molecules of common molecular diameters. The effect of micro-channels is doubled by applying specular reflection at the longitudinal centerlines of channel $\mathrm{F}$ and $\mathrm{R}$. The values of $p_{\mathrm{F}} / p_{\mathrm{R}}$, $p_{\mathrm{D}} / p_{\mathrm{R}}$ and $\delta$ introduced in the previous section are, respectively, $p_{\mathrm{F}} / p_{\mathrm{R}}=1.329, p_{\mathrm{D}} / p_{\mathrm{R}}=0.190$, and $\delta=0.355$. The Knudsen number $\mathrm{Kn}=0.511$ and aspect ratio $t / d=20$. This figure shows the values of mole percentage $\chi$ of smaller gas A by the color map, and the flow velocity $\left(v_{1}, v_{2}\right)$ of mixture by the arrows. The feed gas enters the channel $\mathrm{F}$ at $X_{1} / d=-60$ and flows in $X_{1}$ direction due to the pressure gradient in channel $\mathrm{F}$ to arrive the micro-channels in the membrane at $0<X_{1} / d<100$. In this channel the mole percentage $\chi$ increases as $X_{1}$, since the smaller molecules $\mathrm{A}$ are emitted and larger molecules B are absorbed through the micro-channels. It is also observed that the value of $\chi$ is approximately constant in $X_{2}$ direction. In channel $\mathrm{R}$, the gas enters the system at $X_{1} / d=160$, flows in $-X_{1}$ direction, and is mixed with the outflows of the micro-channels. The effect of membrane is the opposite to that in channel F: the smaller molecules A are absorbed and larger molecules B are emitted through the micro-channels. Therefore, the mole percentage in $\mathrm{R}$ decreases as $X_{1}$ decreases. As the results, $\partial \chi / \partial X_{1}$ are positive in both of channel $\mathrm{F}$ and $\mathrm{R}$. This is important to keep the small size of the difference of mole percentage between two sides of the membrane, which enables us to increase the number of micro-channels to obtain larger variation of $\chi$ along the membrane. The result shown in Fig. 3 demonstrate that this strategy is feasible in principle. The DSMC is not, however, favorable method to calculate the cases for real membrane materials, which may have $10^{4}$ micro-channels in every millimeter, and the 


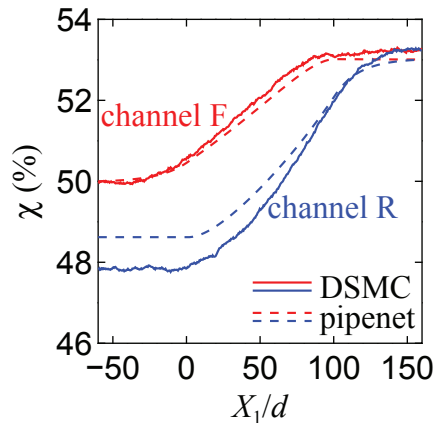

(a)

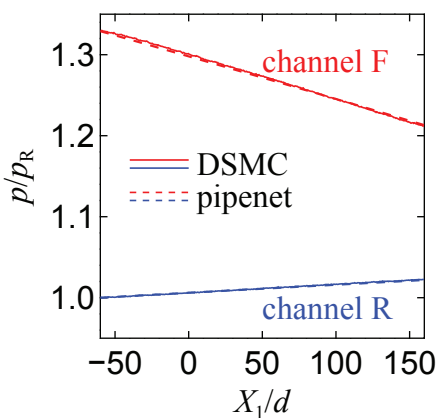

(b)

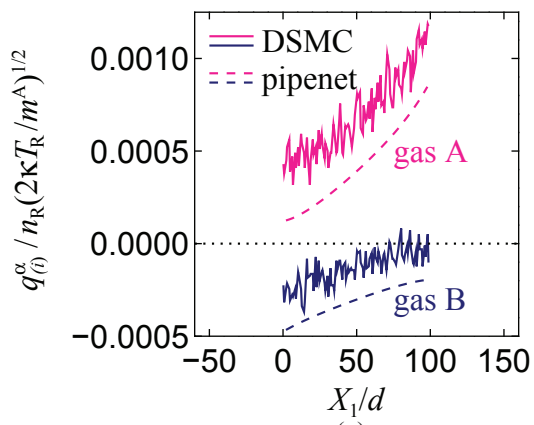

(c)

FIGURE 4. Comparison of DSMC result and that of pipenet method. The case shown in Fig. 3. DSMC result is shown by solid lines, and that of pipenet method is shown by dashed lines. (a) Mole percentage $\chi$ and (b) pressure in channels $\mathrm{F}$ (red) and $\mathrm{R}$ (blue). (c) The molecular flow rate $q_{(i)}^{\alpha}$ of $\alpha$-gas through the $i$-th micro channel. The values are plotted at the position $X_{1}$ of corresponding micro-channel. The difference between DSMC and pipenet results may be difficult to see in panel (b).

computation time for $m=50$ is around few months by several PC with recent Core-i7 CPU. This is the reason why we introduce the pipenet method, which results are described below.

The case presented in Fig. 3 is analyzed by the pipenet method and its steady result is compared with that of DSMC method in Fig. 4. Panel (a) shows the profiles of $\chi$ averaged over the cross sections of channel F(red) and R(blue) respectively. Panel (b) shows that for pressure $p$. The molecular flow rates $q_{(i)}^{\alpha}$ of $\alpha$-gas through $i$-th micro-channel are plotted versus the position $X_{1}$ of the micro-channel in panel (c). The differences $\Delta \chi\left(X_{1}\right)$ and $\Delta p\left(X_{1}\right)$ defined by

$$
\Delta h\left(X_{1}\right)=h\left(X_{1} \text {; channel } \mathrm{F}\right)-h\left(X_{1} \text {; channel } \mathrm{R}\right), \quad(h=\text { cross sectional average of } \chi \text { and } p)
$$

and thus the values of $q_{(i)}^{\alpha}$ are not uniform in $X_{1}$. Despite the small computational time (few seconds), the pipenet method reproduces the qualitative features of the profiles of $\chi$, $p$, etc. and $q_{(i)}^{\alpha}$ of DSMC simulation. Judging from this result, we will estimate the performance of the gas separator with $T_{\mathrm{F}} / T_{\mathrm{R}} \approx 1$ and large $m$ in the following sections.

\section{Effect of the Temperature Ratio}

First of all, we will discuss the effect of the temperature ratio $T_{\mathrm{F}} / T_{\mathrm{R}}$. Figure 5 shows the results of pipenet method for $T_{\mathrm{F}} / T_{\mathrm{R}}=0.9,1.0$, and 1.1. These are the cases for $\mathrm{m}^{\mathrm{B}} / \mathrm{m}^{\mathrm{A}}=2$ of hard-sphere gas with a common diameter of the molecules, $\chi_{\mathrm{F}}=50 \%, \mathrm{Kn}=0.5, t / d=100, D / d=2000$ and the production rate $\delta=0$. The values of pressure ratios $p_{\mathrm{F}} / p_{\mathrm{R}}$ and $p_{\mathrm{D}} / p_{\mathrm{R}}$ are chosen so that the flow velocity $v_{\mathrm{F}}$ in $X_{1}$ direction calculated from molecular flux $N_{\mathrm{F}}$ satisfies $v_{\mathrm{F}} \approx 2 \times 10^{-3}\left(2 \kappa T_{\mathrm{R}} / m^{\mathrm{A}}\right)^{1 / 2}$, and depend on the case. The net molecular exchange condition $Q^{\mathrm{A}}+Q^{\mathrm{B}}=0$ is available at $T_{\mathrm{F}} / T_{\mathrm{R}}=1.1$, where $Q^{\alpha}(\alpha=\mathrm{A}, \mathrm{B})$ are the net molecular flows of $\alpha$-gas through micro-channels

$$
Q^{\alpha}=d \sum_{i=1}^{m} q_{(i)}^{\alpha}
$$

These two conditions for $v_{\mathrm{F}}$ and $Q^{\mathrm{A}}+Q^{\mathrm{B}}=0$ determines $\left(p_{\mathrm{F}} / p_{\mathrm{R}}, p_{\mathrm{D}} / p_{\mathrm{R}}\right)$. Incidentally, the condition $N_{\mathrm{F}}=N_{\mathrm{FL}}$ implies $Q^{\mathrm{A}}+Q^{\mathrm{B}}=0$ from the mass conservation. For other cases, $T_{\mathrm{F}} / T_{\mathrm{R}}=0.9$ and 1 , the solution is parameterized by $p_{\mathrm{D}} / p_{\mathrm{R}}$, thus we plot two representative results in Fig. 5 for each temperature ratio. The number of micro-channels $m$ is $8 \times 10^{4}$ (for $T_{\mathrm{F}} / T_{\mathrm{R}}=1.0$ and 1.1$)$ or $2 \times 10^{4}\left(\right.$ for $\left.T_{\mathrm{F}} / T_{\mathrm{R}}=0.9\right)$. Figure $5(\mathrm{a})$ shows the distribution of $\chi$ in channel $\mathrm{F}$. The value $\chi$ in channel $\mathrm{R}$ is close to that of $\mathrm{F}$ and omitted in the figure [cf. Fig. 8(b)]. As we have seen in Fig. 4, the value of $\chi$ increases with $X_{1}$ for $T_{\mathrm{F}} / T_{\mathrm{R}}>1$. On the other hand, the value of $\chi$ decreases with $X_{1}$ for $T_{\mathrm{F}} / T_{\mathrm{R}} \leq 1$ as is estimated with Fig. 1(c). These features can also be confirmed by separate DSMC calculations, but we omit these results to save space. Figures $5(\mathrm{~b})$ and (c) show, respectively, the flow velocity $v_{1}\left(X_{1}\right)$ calculated from the molecular flow rate in $X_{1}$ direction in channel $\mathrm{F}$ and the pressure difference $\Delta p\left(X_{1}\right)$. For the cases with $T_{\mathrm{F}} / T_{\mathrm{R}}=0.9$ and 1 , the molecules flow in the direction of $\mathrm{F} \rightarrow \mathrm{R}$ through the micro-channels, hence the flow velocity $v_{1}$ decreases with $X_{1}$. The value of $v_{1}$ decreases faster for larger pressure drop $p_{\mathrm{D}} / p_{\mathrm{R}}$. For the cases labeled by $p_{\mathrm{D}}=\max$ in the figure, the 


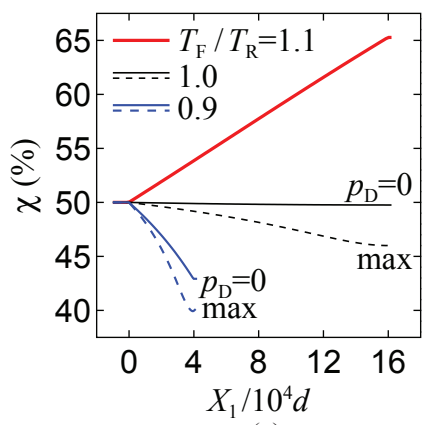

(a)

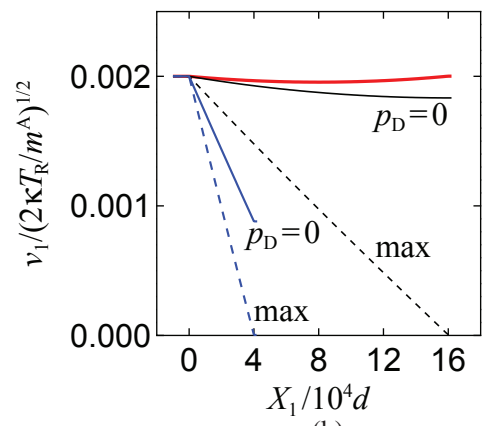

(b)

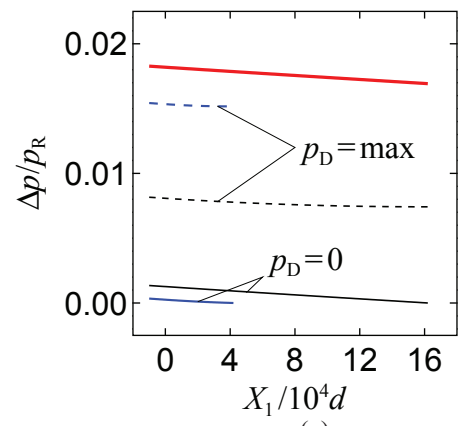

(c)

FIGURE 5. The effect of temperature ratio $T_{\mathrm{F}} / T_{\mathrm{R}}$. The results of pipenet method. The case for $m=8 \times 10^{4}$ (for $T_{\mathrm{F}} / T_{\mathrm{R}}=1.0$ and 1.1) and $2 \times 10^{4}$ (for $T_{\mathrm{F}} / T_{\mathrm{R}}=0.9$ ). The case for hard-sphere mixture with $\mathrm{m}^{\mathrm{B}} / \mathrm{m}^{\mathrm{A}}=2 . \chi_{\mathrm{F}}=50 \%, \mathrm{Kn}=0.5, t / d=100$, $D / d=2000, v_{\mathrm{F}} \approx 2 \times 10^{-3}\left(2 \kappa T_{\mathrm{R}} / \mathrm{m}^{\mathrm{A}}\right)^{1 / 2}$, and production rate $\delta=0$. (a) Mole percentage $\chi$ in channel F; (b) the flow velocity $v_{1}$ in channel F; (c) the pressure difference $\Delta p\left(X_{1}\right)$ between the front and rear sides of the membrane. Two results with different values of $p_{\mathrm{D}}$ are shown for $T_{\mathrm{F}} / T_{\mathrm{R}} \leq 1: p_{\mathrm{D}}=0$ (solid lines) and $p_{\mathrm{D}}=\max$ (dashed lines).

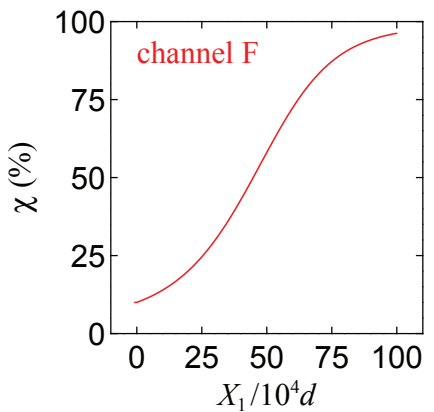

(a)

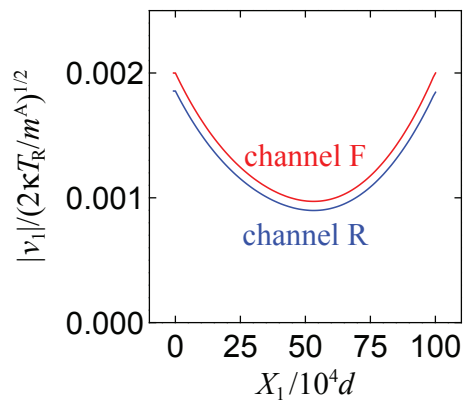

(b)

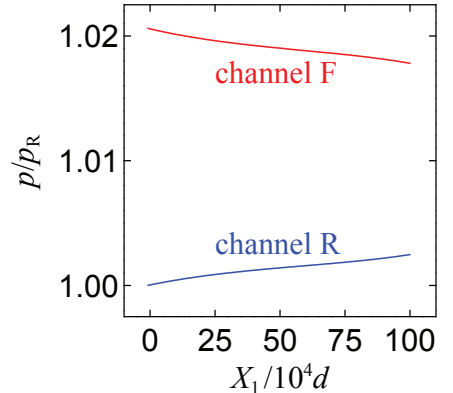

(c)

FIGURE 6. Result of pipenet method for $m=5 \times 10^{5}$ and $\chi_{\mathrm{F}}=10 \%$. The case for $T_{\mathrm{F}} / T_{\mathrm{R}}=1.1$. See the caption of Fig. 5 for other parameters. (a) Mole percentage $\chi$ in channel F. (b) the flow speed $\left|v_{1}\right|$ and (c) pressure $p$ in channels F and R.

value of $v_{1}$ vanishes at the end of channel[Fig. 5(b)]. The value of $\Delta p\left(X_{1}\right)$ naturally decreases with $X_{1}$, and it vanishes at the end of channel if $p_{\mathrm{D}}=0$. In both cases, $p_{\mathrm{D}}=0$ and max, it is impossible to extend the membrane to obtain larger variation of the mole fraction. We are not free from the drop of the flow velocity $v_{1}$ for intermediate values of $p_{\mathrm{D}}$. On the other hand, in the case of $T_{\mathrm{F}} / T_{\mathrm{R}}=1.1$, the flow velocity $v_{1}$ does not vanish due to the molecular exchange condition $Q^{\mathrm{A}}=-Q^{\mathrm{B}}$, and the pressure difference $\Delta p\left(X_{1}\right)$ also does not vanish at $X_{1} \approx L$. This means that we can extend the membrane to obtain larger variation of $\chi$.

The result for larger number of micro-channels $m=5 \times 10^{5}$ is shown in Fig. 6, where the mole percentage of the feed gas is set to $\chi_{\mathrm{F}}=10 \%$. The figure shows the profiles of $\chi$ in channel $\mathrm{F}$ [panel (a)], flow speed $\left|v_{1}\right|$ [panel (b)] and the pressures [panel (c)] in channel F and R. As is seen from Fig. 6(a), the mixture enters channel $\mathrm{F}$ at $\chi_{\mathrm{F}}=10 \%$ and exits $\mathrm{F}$ with $\chi_{\mathrm{FL}} \approx 96 \%$. Figure 6 (b) shows that flow speed in $X_{1}$ direction does not vanish in the device, and Fig. 6(c) shows the pressure difference $\Delta p\left(X_{1}\right)$ also does not vanish even for this large number of micro-channels. The value of $\Delta p\left(X_{1}\right)$, however, depends much on $X_{1}$. The value of $\Delta p\left(X_{1} \approx 0\right)$ is too large for the molecular exchange condition $q_{(i)}^{\mathrm{A}}+q_{(i)}^{\mathrm{B}}=0$ for micro-channels there, and the molecules flow through the micro-channels in the direction of $\mathrm{F} \rightarrow \mathrm{R}$. On the contrary the value of $\Delta p\left(X_{1} \approx L\right)$ is too small and molecules flows $\mathrm{R} \rightarrow \mathrm{F}$. This consideration explains the convex profile of $\left|v_{1}\right|$ seen in panel (b). Incidentally, separate calculations show that $\chi_{\mathrm{FL}} \approx 99.98 \%$ for $\chi_{\mathrm{F}}=95 \%$, and $\chi_{\mathrm{FL}} \approx 12.5 \%$ for $\chi_{\mathrm{F}}=0.1 \%$; we do not find any restriction for mole fraction for this device.

\section{Effect of Flow Speed over the Membrane}

One of the essential phenomena which enables the present device is the convection in channel $\mathrm{F}$ and $\mathrm{R}$. If there were no convection effects, the outflows of the micro-channels in channel $\mathrm{F}$ and $\mathrm{R}$ may diffuse upstream and downstream 


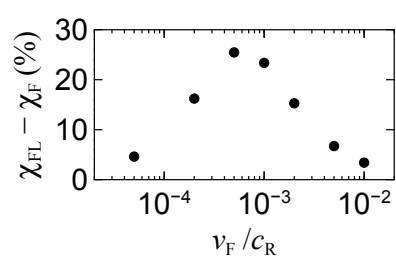

(a)

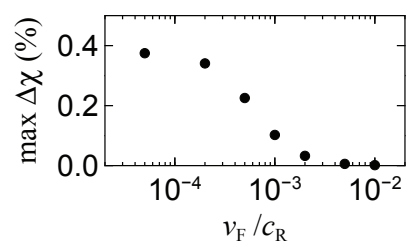

(b)

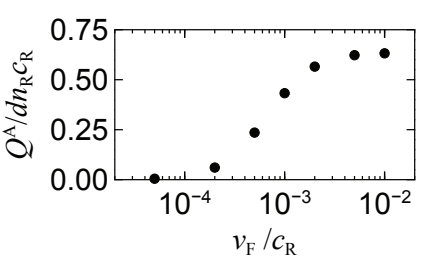

(c)

FIGURE 7. The effect of flow speed $v_{\mathrm{F}}$ at the inlet. The case for $T_{\mathrm{F}} / T_{\mathrm{R}}=1.1$ shown in Fig. 5. (a) The variation of mole percentage $\chi$ along the membrane $\chi_{\mathrm{FL}}-\chi_{\mathrm{F}}$; (b) maximum difference $\max _{0<X_{1}<L} \Delta \chi\left(X_{1}\right)$ of mole percentage between the front and rear sides of membrane; (c) net molecular flux of A-gas through the membrane $Q^{\mathrm{A}} \cdot c_{\mathrm{R}}=\left(2 \kappa T_{\mathrm{R}} / m^{\mathrm{A}}\right)^{1 / 2}$.

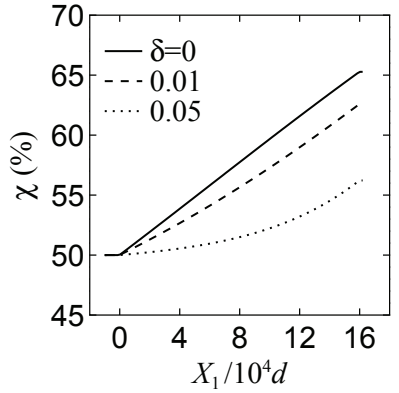

(a)

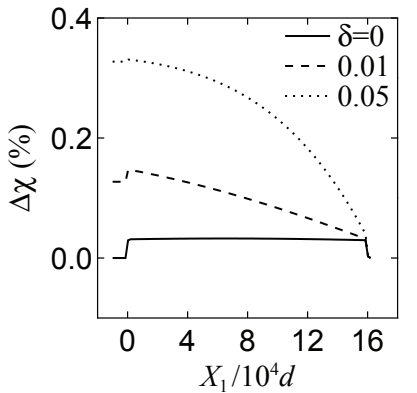

(b)

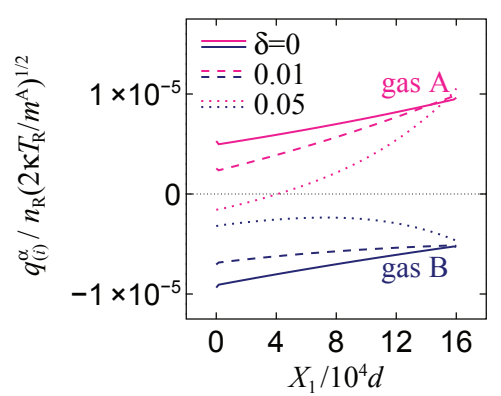

(c)

FIGURE 8. The effect of production rate $\delta$. The case for $T_{\mathrm{F}} / T_{\mathrm{R}}=1.1$ shown in Fig. 5. (a) Mole percentage in channel F; (b) difference of mole percentage between the front and rear sides of membrane; (c) the molecular flow rate $q_{(i)}^{\alpha}$ of $\alpha$-gas through the $i$-th micro channel. The values are plotted at the position of micro-channel.

without distinction. Here we analyze the effect of the flow speed of the feed gas $v_{\mathrm{F}}$, which determines the overall flow speeds in $\mathrm{F}$ and $\mathrm{R}$. We select the case with $T_{\mathrm{F}} / T_{\mathrm{R}}=1.1$ shown in Fig. 5 and carry out the pipenet analysis for various values of $v_{\mathrm{F}}$ by choosing the parameters of the pressure $\left(p_{\mathrm{F}} / p_{\mathrm{R}}, p_{\mathrm{D}} / p_{\mathrm{R}}\right)$ under the condition $Q^{\mathrm{A}}+Q^{\mathrm{B}}=0$. The value of $\chi_{\mathrm{FL}}-\chi_{\mathrm{F}}$, the difference of mole percentage obtained by the membrane, is plotted in Fig. 7(a) versus $v_{\mathrm{F}}$. The value of $\chi_{\mathrm{FL}}-\chi_{\mathrm{F}}$ takes its maximum value at $v_{\mathrm{F}} \approx 10^{-3}\left(2 \kappa T_{\mathrm{R}} / \mathrm{m}^{\mathrm{A}}\right)^{1 / 2}$, and decreases for smaller or larger values of $\nu_{\mathrm{F}}$. For smaller values of $v_{\mathrm{F}}$, the effect of diffusion prevails in channel $\mathrm{F}$ and $\mathrm{R}$; the outflows from the micro-channels stay there. As the results, the difference of mole percentage between the membrane $\Delta \chi\left(X_{1}\right)$, which maximum value $\left[\max _{0<X_{1}<L} \Delta \chi\left(X_{1}\right)\right]$ is plotted in Fig. 7(b), increases. This $\Delta \chi\left(X_{1}\right)$ blocks the molecular exchange flow in the microchannels, and the amount $Q^{\mathrm{A}}$ of their fluxes vanish in the limit of $v_{\mathrm{F}} \rightarrow 0$ [Fig. 7(c)]. For larger values of $v_{\mathrm{F}}$, the value of $Q^{\mathrm{A}}$ increases; that is, larger number of molecules is exchanged by the membrane. The size of $Q^{\mathrm{A}}$ is, however, naturally limited. If the value of $v_{\mathrm{F}}$ is too large, the effect of $Q^{\mathrm{A}}$ is diluted and the variation $\chi_{\mathrm{FL}}-\chi_{\mathrm{F}}$ of mole percentage become small. It is also noted that a side effect of large $v_{\mathrm{F}}$ is the large variation of the pressure in $X_{1}$ direction, which makes difficult to extend the membrane to obtain larger variation $\chi_{\mathrm{FL}}-\chi_{\mathrm{F}}$ of mole fraction. For example, in the case for $v_{\mathrm{F}} /\left(2 \kappa T_{\mathrm{R}} / \mathrm{m}^{\mathrm{A}}\right)^{1 / 2}=0.01$, the value of $\Delta p\left(X_{1}=L\right)$ is around $70 \%$ of that of $\Delta p\left(X_{1}=0\right)$.

\section{Effect of Production Rate}

Finally we will discuss the effect of production rate $\delta$, which is set to zero in the preceding results except the case of Fig. 3. Here we again select the case with $T_{\mathrm{F}} / T_{\mathrm{R}}=1.1$ shown in Fig. 5, and carry out the pipenet method for several values of $\delta$. The results for $\delta=0,0.01$, and 0.05 are compared in Fig. 8. The panel (a) shows the distribution of $\chi$ in channel F. The variation of mole percentage $\chi$ decreases much by the small values of $\delta$. This is inevitable by the present single-membrane construction of the device shown in Fig. 1: If we remove the concentrated gas as the product, the mole percentage at the exit of channel $\mathrm{R}$ decreases from $\chi_{\mathrm{F}}$, but the present device works only for small values of $\Delta \chi\left(X_{1}\right)$. The impact of $\delta$ on the difference $\Delta \chi\left(X_{1}\right)$ is shown in Fig. 8(b). The $\Delta \chi\left(X_{1}\right)$ increases as $\delta$ increases, except the larger $X_{1}$ end where two channels $\mathrm{F}$ and $\mathrm{R}$ are connected. Figure $8(\mathrm{c})$ shows the values of $q_{(i)}^{\alpha}(\alpha=\mathrm{A}, \mathrm{B})$ of $i$-th micro-channel versus the position of the micro-channel. As $\delta$ increases, the value of $q_{(i)}^{\mathrm{A}}-q_{(i)}^{\mathrm{B}}$ decreases, especially at 
$X_{1} \approx 0$. This is the effect of large value of $\Delta \chi\left(X_{1}\right)$ there.

\section{DISCUSSION}

We have analyzed the behavior of the gas mixture in the gas separator proposed in Fig. 1. The DSMC result demonstrates that the gas separator can produce concentrated gas, and the pipenet method is shown to be able to simulate the DSMC result with a reasonable computational cost. The results of the pipenet method show that unlimited variation of mole fraction can be obtained by the present gas separator, where a large number of micro-channels is used instead of large temperature variation in the membrane. We have also carried out a DSMC simulation for the connection part of channels $\mathrm{F}$ and $\mathrm{R}$, and several pipenet calculations for different mass ratios $\mathrm{m}^{\mathrm{B}} / \mathrm{m}^{\mathrm{A}}$, Lennard-Jones intermolecular potential, different values of $t / d$ or Knudsen numbers in micro-channels and channel $\mathrm{F}$ and $\mathrm{R}$. All of these results, which are omitted to save space, support the present design of the gas separator.

The case for $T_{\mathrm{F}} / T_{\mathrm{R}}=1$, where there is no thermal transpiration, corresponds to the membrane gas separator with Knudsen diffusion. In this case the device remains simple without heat source, but its physical performance to obtain the variation of mole fraction is limited. This is the reason why the different mechanism, solution diffusion, is extensively studied in connection with the membrane gas separation [10]. A heat source is required for the case of $T_{\mathrm{F}} / T_{\mathrm{R}}>1$, but it is possible to obtain unlimited variation of mole fraction by a single membrane with no special chemical properties on the material. Small values of production rate $\delta$, however, may reduce the variation of the mole fraction. This is not characteristic to the present method of gas separation, and it will be removed by introducing the cascade connection of units and some reflux process of residual, which are widely used in other methods of gas separation. The estimation of the performance of such systems is left for the future work.

The present results show the possibility to obtain large variation of mole fraction with small temperature variation in a membrane, but further investigation is still required to obtain reliable quantitative estimate. One of the reason is that the numerical methods applied in this paper are the newly proposed pipenet method and the basic DSMC method which is too intensive for slow flows. Another reason is that the present results are based on 2D model even in the microchannels. The flow speeds in $3 \mathrm{D}$ channel at $\mathrm{Kn} \approx 1$ are naturally smaller than those in $2 \mathrm{D}$ channel, and the variation of mole fraction obtained by real device will be different from the present results. One of the common structure of polymeric membranes with sub-micron holes is a fluffy mass of fibers, and the data on the thermal transpiration or diffusion etc. in such structure are required. In view of the structure of the Knudsen pump [1] at atmospheric pressure, channels $\mathrm{F}$ and $\mathrm{R}$ can not be simple channels; they must contain some structure for heat conduction. These differences, however, do not related to the essentials of the present gas separator: the presence of intermediate Knudsen number in micro-channels, and the difference of the dependence on species between the pressure driven flow and the thermal transpiration flow. Therefore, it is expected that this system works after some optimization of parameters, and that the membrane gas separation can be enhanced by the small temperature variation in the membrane.

\section{ACKNOWLEDGMENTS}

The support from Japan Society for the Promotion of Science (Grants-in-Aid \#23560196) is gratefully acknowledged.

\section{REFERENCES}

1. $\quad$ N. K. Gupta and Y. B. Gianchandani, Micropor. Mesopor. Mat. 142, 535-541 (2011).

2. M. Ulbricht, Polymer 47, 2217-2262 (2006).

3. H. Sugimoto, S. Takata, and S. Kosuge, in Rarefied Gas Dynamics, ed. by M. K. Ivanov and A. K. Rebrov, Siberian Branch, Russ. Acad. Sci., 2007, pp. 1158-1164.

4. S. Takata, H. Sugimoto, and S. Kosuge, Eur. J. Mech. B/Fluids 26, 155-181 (2007).

5. H. Sugimoto, in Rarefied Gas Dynamics, ed. by T. Abe, AIAA, 2009, pp. 1123-1128.

6. H. Sugimoto and A. Shinotou, in Rarefied Gas Dynamics, ed. by D. A. Levin, I. J. Wysong, and A. L. Garcia, AIAA, 2011, pp. 784-789.

7. G. A. Bird, Molecular Gas Dynamics and the Direct Simulation of Gas Flows, Clarendon, Oxford, 1994.

8. K. Aoki, P. Degond, S. Takata, and H. Yoshida, Phys. Fluids 19, 117103 (2007).

9. S. Kosuge and S. Takata, Eur. J. Mech. B/Fluids 27, 444-465 (2008).

10. R. W. Baker, Ind. Eng. Chem. Res. 41, 1393-1411 (2002). 
Copyright of AIP Conference Proceedings is the property of American Institute of Physics and its content may not be copied or emailed to multiple sites or posted to a listserv without the copyright holder's express written permission. However, users may print, download, or email articles for individual use. 